\title{
ISO-SWS spectra of [WR] planetary nebulae
}

\author{
Ryszard Szczerba \\ N. Copernicus Astronomical Center, Rabiańska 8, 87-100 Toruń, \\ Poland
}

Kevin Volk

Department of Physics \& Astronomy, University of Calgary, Calgary, Alberta, Canada T2N $1 N_{4}$

\author{
Sławomir K. Górny \\ N. Copernicus Astronomical Center, Rabiańska 8, 87-100 Toruń, \\ Poland
}

\begin{abstract}
We have analyzed Infrared Space Observatory Short Wavelength Spectrometer (ISO-SWS) observations for 16 Wolf-Rayet ([WR]) planetary nebulae ( $\mathrm{PNe}$ ) with the aim of identifying the dust features present in this group of objects. We have found that Polycyclic Aromatic Hydrocarbon (PAH) molecular bands (which are present in most of the observed [WR] PNe) are more frequent than crystalline silicate features.
\end{abstract}

\section{Introduction}

One of the most intriguing ISO results was discovery that coexistence of $\mathrm{O}$ rich and $\mathrm{C}$-rich material is much more common than previously believed. A mixed chemistry is somewhat surprising since formation of $\mathrm{CO}$ (one of the first stable molecules) is so efficient that less abundant element $(\mathrm{C}$ or $\mathrm{O}$ ) is probably mostly used. Among sources with mixed chemistry there are (at least some) [WR] PNe. Recent ISO observations have shown that in this group of objects both forms of dust (C-rich: PAHs and O-rich: crystalline silicates) are present (Waters et al. 1998, Cohen et al. 1999). That [WR] planetary nebulae are unusual was known before ISO mission due to discovery of $\mathrm{OH}$ maser emission from one of this source (Vo 1) by Zijlstra et al. (1991) who already then discussed possibility that this star experienced in the recent past a dramatic change in its chemical composition from O- to C-rich. Presently, this scenario seems to be the most favourable. However, another scenario put forward by Cohen et al. (1999) to explain coexistence of $\mathrm{C}$ - and $\mathrm{O}$-based features in [WR] planetary nebulae by means of destruction of the Kuiper belt (or Oort-cloud) like objects seems to be also possible. Note, that similar scenario has been proposed to explain the presence of water vapour in carbon-rich AGB star IRC+10216 (Melnick et al. 2001). Therefore, investigation of $\mathrm{PAH}$ and crystalline silicate features in [WR] PNe should give us some clues on their evolution and on the related chemical processes inside the expelled matter. 


\section{ISO-SWS spectra of $[W R] P N e$}

In the ISO Data Archive there are 25 SWS spectra for 16 [WR] PNe from the list compiled by Górny (2001). As already discussed by Szczerba et al. (2001) at least $75 \%$ of [WR] PNe have PAH features in their ISO-SWS spectra with K 2-16 being the only clear exception. Table 1 summarizes identified solid state features in [WR] PNe. The $33 \mu \mathrm{m}$ feature is one of the resolved components of the broad $30 \mu \mathrm{m}$ feature (Volk et al. 2002). It seems that crystalline silicates are less frequent than PAHs. Our preliminary results from modelling of these spectra showed that silicates should be cold while C-rich dust components should be relatively warm. In addition, dust emission from neutral parts of the envelopes seems to be necessary to explain far infrared part of the ISO data.

Table 1. Main solid state features in WR [PNe]

\begin{tabular}{llccc}
\hline PN name & $\begin{array}{l}\text { spectral } \\
\text { type }\end{array}$ & $\begin{array}{c}\text { UIBs } \\
\text { crystalline } \\
\text { silicates }\end{array}$ & $\begin{array}{c}\text { other } \\
\text { features }\end{array}$ \\
\hline He 3-1333 & [WC 11] & + & + & \\
He 2-113 & [WC 11] & + & + & \\
Vo 1 & [WC 11] & + & + & \\
M 4-18 & [WC 11] & + & $?$ & \\
K 2-16 & [WC 11] & - & $-?$ & \\
SwSt 1 & [WC 10] & + & - & $33 \mu m ?$ \\
BD +30 3639 & [WC 9] & + & + & \\
He 2-142 & [WC 9] & + & $+?$ & \\
He 2-459 & [WC 9] & + & $+?$ & \\
NGC 40 & [WC 8] & + & $-?$ & \\
M 2-43 & [WC 7] & + & - & $33 \mu m$ \\
Cn 1-5 & [WC 7] & $?$ & $?$ & \\
NGC 6369 & [WC 4] & + & $-?$ & $33 \mu m ?$ \\
NGC 5315 & [WC 4] & + & + & \\
PM 1-89 & [WC 4] & + & $?$ & \\
NGC 5189 & [WC 2] & $?$ & $?$ & \\
\hline
\end{tabular}

Acknowledgments. This work was done while I was visiting the University of Calgary. I would like to thank Sun Kwok for his support of my visit. We acknowledge also support from the grant 2.P03D.024.1p01 from the Polish Committee for Scientific Research.

\section{References}

Cohen, M., Barlow, M.J., Sylvester, R.J., et al. 1999, ApJ, 513, L135

Górny, S.K. 2001, Ap\&SS 275, 67

Melnick, G.J., Neufeld, D.A., Ford, K.E.S., et al. 2001, Nature, 412, 160

Szczerba, R, Górny, S.K., Stasinska, G., et al. 2001, Ap\&SS, 275, 113

Waters, L.B.F.M., Beintema, D.A., Zijlstra A.A., et al. 1998, A\&A, 331, L61

Volk, K., Kwok, S., Hrivnak, B., Szczerba, R. 2002, ApJ, in press

Zijlstra, A.A., Gaylard, M.J., te Lintel Hekkert, P., et al. 1991, A\&A, 243, L9 